\title{
Model assessment through data assimilation of realistic data in cardiac electrophysiology
}

\author{
Antoine Gérard ${ }^{1,3,4}$, Annabelle Collin ${ }^{2,3,4}$, Gautier Bureau ${ }^{5,6}$, Philippe \\ Moireau $^{5,6}$, and Yves Coudière ${ }^{1,2,4}$ \\ 1 IHU Liryc, Electrophysiology and Heart Modeling Institute, F-33000 \\ Pessac-Bordeaux, France. \\ 2 Université Bordeaux, IMB UMR 5251, Talence, France, \\ 3 Bordeaux INP, IMB UMR 5251, Talence, France, \\ 4 Inria, Inria Bordeaux - Sud-Ouest, Talence, France, \\ 5 Inria, Université Paris-Saclay, Palaiseau France, \\ 6 LMS, Ecole Polytechnique, CNRS UMR 7649, Institut Polytechnique de Paris, \\ Palaiseau, France
}

\begin{abstract}
We consider a model-based estimation procedure - namely a data assimilation algorithm - of the atrial depolarization state of a subject using data corresponding to electro-anatomical maps. Our objective is to evaluate the sensitivity of such a model-based reconstruction with respect to model choices. The followed data assimilation approach is capable of using electrical activation times to adapt a monodomain model simulation, thanks to an ingenious model-data fitting term inspired from image processing. The resulting simulation smoothes and completes the activation maps when they are spatially incomplete. Moreover, conductivity parameters can also be inferred. The model sensitivity assessment is performed based on synthetic data generated with a validated realistic atria model and then inverted using simpler modeling ingredients. In particular, the impact of the muscle fibers definition and corresponding anisotropic conductivity parameters is studied. Finally, an application of the method to real data is presented, showing promising results.
\end{abstract}

Keywords: Cardiac modeling · electrophysiology $\cdot$ Data assimilation

\section{Introduction}

Data assimilation consists in coupling a dynamical model with available measurements in order to register the model on the data and identify model parameters of interest for a specific diagnosis. This approach is now recognized as a potential key ingredient to personalize computational models on clinical measurements allowing to produce predictive patient specific simulations, in particular in electrophysiology [11|6 13 23]. The purpose of this work is to evaluate the robustness of assimilating electrical catheter data based on such a model-based estimation procedure when relying on different modeling assumptions. To this end, we need a state-of-the-art data assimilation procedure, some controlled or real measurements, and the model options considered. For the data assimilation strategy, we 
choose the sequential approach introduced in [2]. For the available measurements, we generate realistic synthetic datasets, produced by a controlled refined model. For the modeling options, we propose to use two variants of a simpler model: one which does not take into account the fiber direction and one which does. The result of the data assimilation will be in the first case the estimation of an isotropic conductivity tensor without building the fiber distribution of the left atrium. In the second case, two conductivity parameters are identified and the conductivity tensor is reconstructed using the fiber distribution. Eventually, these estimated parameters allow to compute activation times which are compared to the available measurements. Finally, we propose a first attempt of the data assimilation procedure on a real patient's dataset from RHYTHMIA HDx ${ }^{\mathrm{TM}}$ recording. Here, five anatomical regions are defined on the electro-anatomical recording mesh and an isotropic conductivity tensor is estimated in each region. This example allows to understand the remaining limitations of the procedure in a clinical context.

\section{Problem setting and estimation methodology}

\subsection{Models formulation}

Model used to generate realistic synthetic data Realistic synthetic data are computed as solutions to the bilayer atrial model defined in [7]: transmembrane voltages are defined on two layers of the atrial surface coupled in a resistive manner, but with independent ionic currents and fiber directions. They may be seen as the endo- and epicardial layers, and the two transmembrane voltages $u^{(k)}(k=1,2)$ solve the following monodomain equations with the coupling coefficient $\gamma>0$ :

$$
\partial_{t} u^{(k)}+\frac{I_{\mathrm{ion}, k}}{C_{m}}\left(u^{(k)}, \cdot\right)=\operatorname{div}\left(\frac{\sigma^{(k)}}{\xi_{m} C_{m}} \nabla u^{(k)}\right)+(-1)^{k} \gamma\left(u^{(1)}-u^{(2)}\right),
$$

for all $t>0, x \in \Omega$ where $\Omega$ is the atrial surface. The ratio $\xi_{m}$ of surface of membrane per unit volume of tissue and the membrane capacitance $C_{m}$ are fixed. The conductivity tensors $\sigma^{(k)}(x)$ can be different on each layer. To obtain realistic simulations, we consider - like in [7] - two different conductivity coefficients defined from endo- and epicardial fiber directions as $\sigma^{(k)}(x)=$ $\sum_{i=1}^{2} d_{i} \nu_{i}^{(k)}(x) \nu_{i}^{(k)}(x)^{T}$, where $\left(\nu_{1}^{(k)}, \nu_{2}^{(k)}\right)$ define the local directions parallel and perpendicular to the fiber on the surface $\Omega$. The values of the conductivity parameters $d_{i}$ are piecewise constant on a few subdomains of the atria allowing to considerer specific atrial structures as sinus node, Bachmann bundle and pectinate muscles. Concerning the ionic model given by the functions $I_{\text {ion, } k}$, the CRN [4] ionic model (16 gating variables and 5 ion concentrations) is considered on each of the two layers.

Model used for the estimation To study the robustness of the data assimilation strategy faced with modeling errors, the model used for the estimation 
is simplified in several ways. Indeed, instead of having a two layers model with local electrophysiological parameters, as the one used to create data, we will consider only one layer

$$
\partial_{t} u+\frac{I_{\text {ion }}}{C_{m}}(u, \cdot)=\operatorname{div}(d \nabla u), \quad t>0, x \in \Omega .
$$

The equation is a simplified version of the bilayer model (1) in which only the (endocardial) transmembrane voltage $u$ is considered. It is coupled with the much simpler Mitchell-Schaeffer ionic model (MS) [8] (compared to the CRN model). Furthermore, the conductivity tensor $d$ is assumed to be homogeneous (i.e. specific atrial structures are not considered). Moreover, in order to analyze the importance of knowing the fiber distribution, we will compare results of two data assimilation models: one in which fiber distribution is unknown (isotropic), i.e. $d:=d_{0} \mathrm{Id}$ and the other where it is known (anisotropic), i.e. $d:=\sum_{i=1}^{2} d_{i} \nu_{i}^{(1)}(x) \nu_{i}^{(1)}(x)^{T}$ (defined by the endocardial fiber direction from the realistic model described above).

\subsection{Presentation of the estimation method}

Our objective is to complete and regularize the activation maps recorded by clinical catheter systems, and to adjust the conductivity tensor $d$ in Eq. (2) based only on the observed activation maps information. Indeed, our data are the values of the activation map $0 \leq t_{a}(x) \leq T$, where $t_{a}(x)$ is the time of first arrival of the electrical activation at $x \in \Omega$. It also defines our working time interval $[0, T]$. From a given data $t_{a}$, we introduce the following activation function

$$
z(x, t)=\left\{\begin{array}{ccc}
1 & \text { if } t>t_{a}(x) \quad \text { (activated region) } \\
-1 & \text { if } t<t_{a}(x) \quad \text { (region at rest) }
\end{array}\right.
$$

This new quantity can then be compared to the solution $u$ of the model (Eq. 22) by defining the activated region $\Omega_{u}^{+}(t):=\left\{x ; u(x, t)>u_{\mathrm{th}}\right\}$, the region at rest $\Omega_{u}^{-}(t):=\left\{x ; u(x, t)<u_{\mathrm{th}}\right\}$, and the activation front $\Gamma_{u}(t)=\left\{x ; u(x, t)=u_{\mathrm{th}}\right\}$ (here $u_{\mathrm{th}}>$ is the given activation threshold). The discrepancy $D$ between the solution $u$ and the observation $z$ (or $t_{a}$ ), a key ingredient of the estimation strategy, is then given by

$$
D(z, u)=\left(1-H\left(u-u_{\mathrm{th}}\right)\right)\left(z-c^{-}(z, u)\right)+H\left(u-u_{\mathrm{th}}\right)\left(z-c^{+}(z, u)\right),
$$

where $H$ is the Heaviside function and $c^{ \pm}(z, u)=\frac{\int_{\Omega_{u}^{ \pm}} z(x, t) d x}{\left|\Omega_{u}^{ \pm}\right|}$. The objective is that (1) $D=0$ when the model is registered on the data and (2) the sensitivity of $D$ to $u$ and $z$ allows to find a direction of model correction when model and data do not coincide.

State observer In order to take into account the modeling errors and the initial condition uncertainties, we first introduce a corrected dynamics

$$
\partial_{t} u+\frac{I_{\text {ion }}}{C_{m}}(u, w)=\operatorname{div}(d \nabla u)+\mathcal{L}(z, u), \quad t \in[0, T], \quad x \in \Omega,
$$


with $u(0, x)=u_{0}(x)$ any arbitrary initial data. The correction filter $\mathcal{L}(z, u)-$ initially introduced in [2] - is directly inferred from the discrepancy $D$ sensitivity with respect to the front evolution:

$$
\mathcal{L}(z, u)=\lambda \delta_{u-u_{\mathrm{th}}}\left(\left(z-c^{-}(z, u)\right)^{2}-\left(z-c^{+}(z, u)\right)^{2}\right),
$$

where $\lambda>0$ is the gain parameter (and can be related to the data confidence) and $\delta_{u-u_{\mathrm{th}}}$, the Dirac distribution on the surface $\Gamma_{u}(t)$. As wished, the filter effect vanishes when simulated observations and recorded observations coincide.

Reduced order joint state and parameter filtering As we also want to estimate the conductivity tensor $d$, we follow the strategy introduced in [9]: the state $u$ is corrected with the state observer (5) whereas the conductivity tensor $d$ is estimated with a reduced Kalman filter. We actually use a Reducedorder Unscented Kalman Filter (RoUKF) [10], implemented in the Verdandi library [1. Moreover, the parameter estimation is realized following an iterative Kalman filter strategy [5] which consists in several consecutive estimations using the previous estimated parameters as an a priori for the next one.

\section{Synthetic and clinical data}

\subsection{Computation of realistic synthetic data}

We compute realistic activation times based on solutions of the bilayer model recalled in Section 2.1, and detailed in [7. The equations are discretized on two coupled meshes of the endo- and epicardial layers of the right and left atria (total of 348657 vertices and 690117 triangles). Each layer has its own fiber distribution, and regional electrophysiology with 13 different regions, e.g. the CRN ionic model is tuned to have a short APD in the pulmonary veins, inactive regions around the sinus node, fast propagation in the Bachmann bundle, specific properties in the pectinate muscles.

Here, we simulate numerically three different catheter pacing scenarii. We pace the sinus node at $1.33 \mathrm{~Hz}$ in order to mimic the sinus rhythm, and, after $1.6 \mathrm{~s}$ of free running sinus rhythm:

LIPV: pace from a location in the left inferior pulmonary vein at $3.33 \mathrm{~Hz}$;

LAR: pace from a location in the roof of the left atrium at $3.33 \mathrm{~Hz}$;

LAA: pace from a location in the appendage of the left atrium at $3.33 \mathrm{~Hz}$.

Each simulation runs for a total time of $5 \mathrm{~s}$, which results in successive focal activations of the left atria. In each case, a unique activation time map $t_{a}(x)$ is computed from an average of these activations. At last, the activation maps are projected on a coarse mesh of 20773 nodes and 41129 triangles. 


\subsection{Clinical data}

The clinical dataset (Fig. 1) consists in a left atrium electro-anatomical map acquired on a patient suffering from atrial tachycardia, using the RHYTHMIA $\mathrm{HDx}^{\mathrm{TM}}$ system. The mesh contains 10140 nodes and 20032 triangles.

\section{Results and discussion}

\subsection{Synthetic data}

Computation of local activation times, and total activation duration. The estimation of the conductivity $d_{0}$ (resp. $\left(d_{1}, d_{2}\right)$ ) is realized using the iterative strategy with five consecutive simulations from an initial guess $d_{0}=5 \mathrm{~S} \mathrm{~cm}^{-1}$ (resp. $\left.\left(d_{1}, d_{2}\right)=(5,5)\right)$. Each of the five estimated parameters (resp. 10) is used to reconstruct an activation map using the classical monodomain model presented in Section 2.1. Modeled activation times will be denoted by $\overline{t_{a}}(x)$ and computed as $\overline{t_{a}}(x)=\min \left\{t \mid u(x, t) \geq u_{t h}\right\}$. Then, these computed activation times are compared with the data activation times $t_{a}(x)$. In Table 1 , we display the evolution of the estimated parameters along iteration of the iterative Kalman filter as well as the relative error - in percent - between total activation duration for both data and reconstructed times. This total activation duration is nothing else than the range of $t_{a}(x)\left(\operatorname{resp} . \overline{t_{a}}(x)\right)$. If we denote by $T A D$ the total activation duration of the dataset and $\overline{T A D}$ the modeled one, the "error" entry in Table 1 is computed as $\frac{|T A D-\overline{T A D}|}{T A D} \times 100$. In Table 2 we expose the minimum and maximum of the pointwise difference $t_{a}(x)-\overline{t_{a}}(x)$ as well as 25 th, 50th and 75th percentiles denoted as Q1, Q2, and Q3.

Convergence of the conductivity coefficients. By looking at the parameter evolutions, we can notice that - contrary to the anisotropic case, - the isotropic case seems to depend on the pacing site. Indeed, at the fifth step $d_{0}$ is enclosed between 2.27 and $4.28 \mathrm{~S} \mathrm{~cm}^{-1}$. Furthermore, it appears that in the isotropic case, $d_{0}$ converges for both LIPV and LAR cases whereas it oscillates in LAA case.

Total activation duration. If we now look at the relative error between total activation duration, LAA is still the worst case with $31.9 \%$ of error at the fifth iteration. For both LIPV and LAR, we decrease the relative error to respectively $4.8 \%$ and $12.7 \%$ after five iterations. The parameter identification process seems to be more efficient when estimating an anisotropic tensor. At the fifth iteration, each of the three couples $\left(d_{1}, d_{2}\right)$ are similar, and we recover an anisotropy ratio which is consistent with the one used to create data. Again, LAA relative error of total activation duration increases in the anisotropic case but stays lower than $6 \%$ over all iterations.

Distribution of local activation times. If we now look at point-wise differences in Table 2, the same conclusion prevails: knowing the fiber distribution is essential to build a model faithful to the observations. For example, point-wise differences 


\begin{tabular}{|c|c|c|c|c|c|c|c|c|c|c|c|}
\hline \multicolumn{6}{|c|}{ Without fiber (isotropic) case } & \multicolumn{6}{|c|}{ With fiber (anisotropic) case } \\
\hline iter. & 1 & 2 & 3 & 4 & 5 & iter. & 1 & 2 & 3 & 4 & 5 \\
\hline \multicolumn{12}{|l|}{$L I P V$} \\
\hline$d_{0}$ & 2.4 & 4.3 & 2.9 & 3.8 & 3.5 & $d_{1}$ & 5.6 & 9.8 & 6.9 & 8.5 & 7.7 \\
\hline- & - & - & - & - & - & $d_{2}$ & 1.3 & 2.4 & 1.9 & 2.1 & 2.0 \\
\hline error $(\%)$ & 14 & 14 & 3.9 & 9.7 & 4.8 & error $(\%)$ & 20 & 8.5 & 3.0 & 2.9 & 0.10 \\
\hline \multicolumn{12}{|l|}{$L A R$} \\
\hline$d_{0}$ & 5.1 & 4.9 & 4.6 & 4.3 & 4.3 & $d_{1}$ & 7.3 & 8.7 & 7.5 & 8.3 & 7.7 \\
\hline- & - & - & - & - & - & $d_{2}$ & 1.5 & 2.3 & 1.9 & 2.1 & 2.0 \\
\hline error (\%) & 19 & 18 & 16 & 13 & 13 & error $(\%)$ & 5.2 & 9.4 & 2.3 & 6.6 & 3.9 \\
\hline \multicolumn{12}{|l|}{$L A A$} \\
\hline$d_{0}$ & 3.0 & 5.6 & 2.3 & 5.6 & 2.3 & $d_{1}$ & 7.3 & 7.7 & 7.6 & 7.6 & 7.7 \\
\hline- & - & - & - & - & - & $d_{2}$ & 2.8 & 2.4 & 2.5 & 2.5 & 2.5 \\
\hline error $(\%)$ & 16 & 14 & 30 & 14 & 32 & error $(\%)$ & 2.8 & 6.3 & 5.6 & 5.8 & 5.8 \\
\hline
\end{tabular}

Table 1: Conductivity coefficients, and relative errors on the activation duration along the iterates.

for LIPV and LAR are respectively enclosed in intervals $[-10,11]$ and $[-11.5,8.8]$ for anisotropic cases, whereas it was in $[-22.8,16]$ and $[-15.4,20.4]$ intervals for isotropic cases. Again, LAA case is less convincing than the others but we can notice an improvement of the three quartiles Q1, Q2 and Q3 for this case when we estimate an anisotropic tensor.

\begin{tabular}{|c|c|c|c|c|c|c|c|c|c|c|c|}
\hline \multicolumn{6}{|c|}{ Without fiber (isotropic) case } & \multicolumn{6}{|c|}{ With fiber (anisotropic) case } \\
\hline Iter. & 1 & 2 & 3 & 4 & 5 & Iter. & 1 & 2 & 3 & 4 & 5 \\
\hline \multicolumn{12}{|c|}{$L I P V$} \\
\hline Min. & -42 & -14 & -32 & -19 & -23 & Min. & -33 & -7.3 & -12 & -8.9 & -10.0 \\
\hline Q1 & -20 & -1.5 & -13 & -4.3 & -6.9 & Q1 & -18 & 1.7 & -5.7 & -1.8 & -3.8 \\
\hline Q2 & -12 & 2.4 & -6.3 & 0.17 & -1.7 & Q2 & -14 & 3.7 & -3.9 & 0.13 & -1.8 \\
\hline Q3 & -5.2 & 8.0 & -0.26 & 5.2 & 3.4 & Q3 & -8.7 & 5.8 & -1.3 & 2.4 & 0.53 \\
\hline Max. & 5.0 & 22 & 10 & 18 & 16 & Max. & 4.0 & 15 & 9.1 & 13 & 11 \\
\hline \multicolumn{12}{|c|}{$L A R$} \\
\hline Min. & -9.7 & -11 & -13 & -15 & -15 & Min. & -16 & -8.1 & -12 & -9.8 & -12 \\
\hline Q1 & -2.3 & -3.0 & -4.2 & -6.0 & -6.2 & Q1 & -4.9 & 2.2 & -1.3 & 1.2 & -0.34 \\
\hline Q2 & 3.5 & 2.9 & 1.8 & 0.38 & 0.24 & Q2 & -1.8 & 3.8 & 0.44 & 2.5 & 1.1 \\
\hline Q3 & 11 & 10 & 9.3 & 8.0 & 7.8 & Q3 & 0.34 & 5.7 & 2.4 & 4.3 & 3.0 \\
\hline Max. & 23 & 23 & 22 & 21 & 20 & Max. & 4.1 & 12 & 7.8 & 11 & 8.8 \\
\hline \multicolumn{12}{|l|}{$L A A$} \\
\hline Min. & -39 & -15 & -52 & -14 & -53 & Min. & -21 & -22 & -22 & -22 & -22 \\
\hline Q1 & -17 & 2.0 & -28 & 2.1 & -29 & Q1 & -2.5 & -3.1 & -2.9 & -3.0 & -3.0 \\
\hline Q2 & -9.8 & 5.0 & -18 & 5.1 & -18 & Q2 & 1.8 & 1.1 & 1.3 & 1.2 & 1.2 \\
\hline Q3 & -3.4 & 8.6 & -8.8 & 8.7 & -9.4 & Q3 & 3.7 & 2.5 & 2.8 & 2.6 & 2.6 \\
\hline Max. & 13 & 25 & 11 & 25 & 11 & Max. & 19 & 19 & 19 & 19 & 19 \\
\hline
\end{tabular}

Table 2: Statistics for $t_{a}(x)-\overline{t_{a}}(x)-\mathrm{Q} 1, \mathrm{Q} 2$ and Q3 respectively represent first, second and third quartiles. 
Importance of fiber structure. These results show us how essential is the fiber distribution in cardiac modeling if we want models to be consistent with observed activation times. Even if we reduce error on total activation duration for two of the three cases in the isotropic context, the range of point-wise differences between activation times stays quite large for each of the three cases. Moreover, the isotropic case gives us three different conductivity parameters $d_{0}$. This is surely due to the difference of anisotropy of paced region but also because data were created using two layers and regional electrophysiological parameters while we are trying to estimate an isotropic conductivity tensor on a one layer model with global electrophysiological parameters. Incidentally, when we take into account the fiber distribution, we are able to reduce the range of the point-wise differences for the three cases. In the anisotropic case, the relative error on total activation duration is enclosed between 0.1 and $5.8 \%$ which is promising. Nevertheless, even though prescribing a fiber architecture allows us to reduce the point-wise differences between activation times, there is still some contrast surely due to model simplification. Indeed, as we already said before, data were created using local parameters whereas we estimate global parameters in order to see if we could build a simpler model which fits at best observed activation times. In this way, estimating an anisotropic tensor seems to be the best strategy and gives us encouraging results.

\subsection{Clinical Data}

Computation on clinical dataset. The same work is carried out on the clinical dataset presented in Section 3.2. For this dataset, fiber distribution is not known, but we have seen in previous section how important it is. We will try to overcome this by defining five different regions in the atria and estimate an isotropic tensor on each one. Those five regions were manually created using the formalism of [12] and can be resumed as: anterior wall, lateral wall, septum, inferior wall and roof.

\begin{tabular}{lrrr}
\hline \multicolumn{4}{c}{ Clinical dataset } \\
Iter. & \multicolumn{1}{c}{1} & \multicolumn{1}{c}{2} & \multicolumn{1}{c}{3} \\
\hline Min & -26.70 & -26.70 & -43.32 \\
Q1 & 0.65 & 2.79 & -3.38 \\
Q2 & 8.35 & 10.90 & 3.37 \\
Q3 & 19.40 & 21.27 & 16.02 \\
Max & 66.26 & 70.46 & 63.55 \\
error (\%) & 19.60 & 7.60 & 1.70 \\
\hline
\end{tabular}

Table 3: Statistics of $t_{a}(x) \overline{-\overline{t_{a}}(x) \text {, and relative error }}$ on the activation duration along the iterates - clinical dataset.

Distribution of local activation times, and total activation duration. As for the synthetic cases, we display in Table 3 the point-wise differences between the 
dataset and the estimated activation map. An additional column gives the relative error of total activation duration. In this real case, only the relative error on activation duration is improved. The point-wise difference between activation times stays large and at the third iteration, we are between -43.32 and $63.55 \mathrm{~ms}$ with $50 \%$ of the data between -3.38 and $16.02 \mathrm{~ms}$.

Anisotropy and measure artefact effect. These differences could be explained in several ways. One of them lies in the apparent strong anisotropy in the data. We emphasized this anisotropy on Fig. 1 by drawing in black the isolines $\left\{t_{a}(x)=t_{i}, t_{i}=10 i, i=0 \ldots 12\right\}$. Therefore, even if we split the atria in several regions, we will not be able to model the anisotropy without prescribing a fiber distribution. Moreover, some measure artefacts like high front slowdowns or accelerations showed on Fig 1 are not reproduced by the model which leads to more error between target and reconstructed times. These slowdowns and acceleration are probably measurement artefacts, due to the electrical signals processing methods that detect the activation.
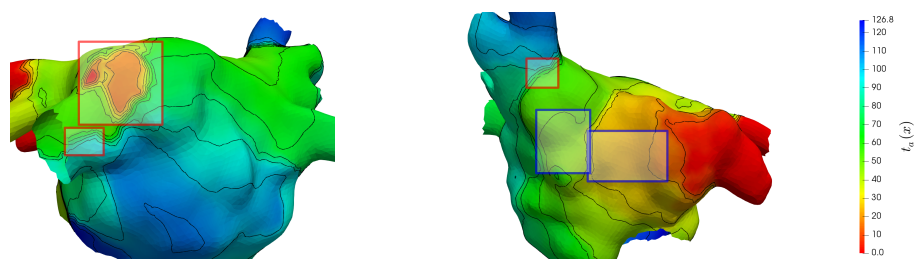

Fig. 1: High front slowdowns (red rectangles) and high accelerations (blue rectangles) on real data isolines.

\section{Conclusion}

In this paper, we assess the robustness of a data assimilation method when facing with modeling simplifications. To do so, we firstly estimate conductivity parameters in two different ways, using realistic synthetic data. The first strategy consists in considering a unique conductivity parameter without taking into account the fiber distribution of the left atrium. This method allows us to decrease the relative error of total activation duration in two cases but was not reliable in the third case. The second one - consisting in estimating two conductivity parameters and using the fiber direction - is promising and allows to recover a large anisotropy ratio in the three cases. This illustrates how much the fiber architecture and the anisotropy of the conductivity are essential.

The application of our strategy on real, but rather noisy, data - which does not contain the fiber architecture - illustrates the sensitivity of the procedure to modeling choices. Moreover, in this case, due to the inherent acquisition and signal processing errors, there is no guarantee that the clinical activation map is 
consistent with the solution of a monodomain model, and hence the data quality is also in question. To conclude, this first attempt paves the way of evaluating modeling choices and data acquisition quality through real data assimilation model-data coupling.

\section{References}

1. Chapelle, D., Fragu, M., Mallet, V., Moireau, P.: Fundamental principles of data assimilation underlying the Verdandi library: applications to biophysical model personalization within euHeart. Medical \& Biological Eng \& Computing pp. 1-13 (2012)

2. Collin, A., Chapelle, D., Moireau, P.: A Luenberger observer for reaction-diffusion models with front position data. Journal of Computational Physics 300, 288-307 (2015)

3. Corrado, C., Whitaker, J., Chubb, H., Williams, S., Wright, M., Gill, J., O'Neill, M.D., Niederer, S.A.: Personalized models of human atrial electrophysiology derived from endocardial electrograms. IEEE Transactions on Biomedical Engineering 64(4), 735-742 (2017)

4. Courtemanche, M., Ramirez, R., Nattel, S.: Ionic mechanisms underlying human atrial action potential properties: insights from a mathematical model. American Journal of Physiology 275, H301-H321 (1998)

5. Jazwinski, A.H.: Stochastic Processes and Filtering Theory. Academic Press (1970)

6. Konukoglu, E., Menze, B.H., Relan, J., Cilingir, U., Chinchapatnam, P., Jadidi, A., Cochet, H., Hocini, M., Delingette, H., Jais, P., Haissaguerre, M., Ayache, N., Sermesant, M.: Efficient probabilistic model personalization integrating uncertainty on data and parameters: Application to Eikonal-Diffusion models in cardiac electrophysiology. Prog Biophys Mol Bio 107(1), 134-146 (2011)

7. Labarthe, S., Bayer, J., Coudière, Y., Henry, J., Cochet, H., Jaïs, P., Vigmond, E.: A bilayer model of human atria: mathematical background, construction, and assessment. Europace 16(suppl 4), iv21-iv29 (2014)

8. Mitchell, C., Schaeffer, D.: A two-current model for the dynamics of cardiac membrane. Bulletin Math. Bio. 65, 767-793 (2003)

9. Moireau, P., Chapelle, D., Le Tallec, P.: Joint state and parameter estimation for distributed mechanical systems. Computer Methods in Applied Mechanics and Engineering 197, 659-677 (2008)

10. Moireau, P., Chapelle, D.: Reduced-order unscented kalman filtering with application to parameter identification in large-dimensional systems. ESAIM: Control, Optimisation and Calculus of Variations 17(2), 380-405 (2011)

11. Moreau-Villeger, V., Delingette, H., Sermesant, M., Ashikaga, H., McVeigh, E., Ayache, N.: Building maps of local apparent conductivity of the epicardium with a 2-D electrophysiological model of the heart. Biomedical Engineering, IEEE Transactions on 53(8), 1457-1466 (2006)

12. Prabhu, S., Voskoboinik, A., McLellan, A.J., Peck, K.Y., Pathik, B., Nalliah, C.J., Wong, G.R., Azzopardi, S.M., Lee, G., Mariani, J., et al.: Biatrial electrical and structural atrial changes in heart failure: electroanatomic mapping in persistent atrial fibrillation in humans. JACC: Clinical Electrophysiology 4(1), 87-96 (2018)

13. Talbot, H., Cotin, S., Razavi, R., Rinaldi, C., Delingette, H.: Personalization of cardiac electrophysiology model using the unscented kalman filtering. In: Computer Assisted Radiology and Surgery (CARS 2015) (2015) 